\title{
SÍNTESE PELO MÉTODO DO PRECURSOR POLIMÉRICO MODIFICADO DA FERRITA DE BISMUTO
}

\section{SYNTHEIS BY MODIFIED POLYMERIC PRECURSOR METHOD OF BISMUTH FERRITE}

A. V. SUAREZ ${ }^{1}$, M. M. TOGASHI ${ }^{1}$; C. F. PERDOMO ${ }^{1}$, R. F. K. GUNNEWIEK ${ }^{2}$ e R. H. G. A. KIMINAMI ${ }^{1}$

${ }^{1}$ Universidade Federal de São Carlos, Departamento de Engenharia de Materiais, Brasil

${ }^{2}$ Universidade Federal de Alfenas, Departamento de Engenharia de Materiais, Brasil

E-mail: arianesuarez@dema.ufscar.br

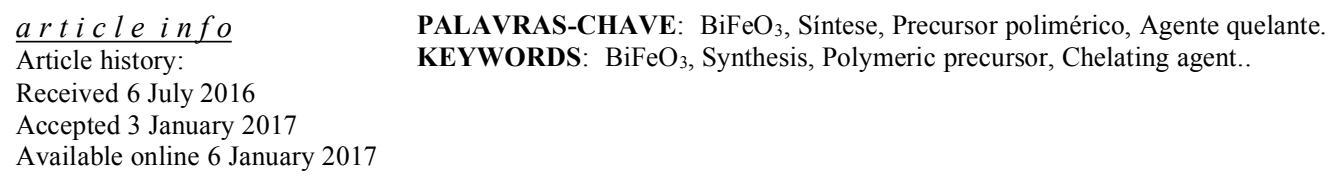

PALAVRAS-ChAVE: $\mathrm{BiFeO}_{3}$, Síntese, Precursor polimérico, Agente quelante. KEYWORDS: $\mathrm{BiFeO}_{3}$, Synthesis, Polymeric precursor, Chelating agent..

RESUMO: Pós de ferrita de bismuto ( $\mathrm{BiFeO}_{3}$ ) foram sintetizados com sucesso mediante o método do precursor polimérico modificado, utilizando como agente quelante, ácido poliacrílico de peso molecular 1800. A morfologia e a estrutura cristalina dos pós obtidos foram analisadas através de difração de raios $X$, área de superficie especifica BET e microscopia eletrônica de varredura (MEV). Os resultados indicaram não apenas a redução de fases secundárias a um valor mínimo de aproximadamente $7 \%$ em $600{ }^{\circ} \mathrm{C}$, mas também um menor tamanho de cristalito de $70 \mathrm{~nm}$. Foram também observadas partículas com morfologia tipo agulha, associada à presença da fase $\mathrm{Bi}_{25} \mathrm{FeO}_{40}$ no sistema, que com o aumento da temperatura foram notavelmente reduzidas. Com menos presença de fases secundárias, a $\mathrm{BiFeO}_{3}$ obtida neste trabalho possui grande potencial em aplicações na indústria de eletroeletrônicos.

\begin{abstract}
Powder of bismuth ferrite ( $\left.\mathrm{BiFeO}_{3}\right)$ were successfully synthesized by modified polymeric method using polyacrylic acid with molecular weight of 1800 as chelating agent. The morphology and crystalline structure of the synthesized powders were analyzed through X-ray diffraction, BET and scanning electron microscopy (SEM). The results indicate not only the reduction of secondary phases to a minimum value of approximately $7 \%$ at $600{ }^{\circ} \mathrm{C}$, but a smallest crystallite size of $70 \mathrm{~nm}$, and particles with elongated forms, directly associated with the increase of secondary phases of $\mathrm{Bi}_{25} \mathrm{FeO}_{40}$. With less presence of secondary phases, $\mathrm{BiFeO}_{3}$ obtained in this work has exciting applications potential in the electronics industry.
\end{abstract}

\section{INTRODUÇÃO}

Um dos materiais representativos dos materiais multiferroicos é a ferrita de bismuto $\left(\mathrm{BiFeO}_{3}\right)$, pertencente à classe dos multiferroicos intrínsecos, que apresenta simultaneamente propriedades magnéticas e ferroelétricas, fazendo deste material um candidato potencial para várias aplicações (ČEBELAA et al., 2017), tais como armazenadores de informação, spintronics, sensores e elementos de memórias de múltiplos estados, nos quais a informação pode ser armazenada tanto nos estados de polarização quanto no estado de magnetização 
espontânea do elemento. Mesmo com vários métodos de obtenção da $\mathrm{BiFeO}_{3}$, é comum o aparecimento de fases secundárias (FISCHER et al., 1980; MAITRE et al., 2004), o que evidencia a complexidade da síntese mesmo usando métodos químicos, permitindo a exploração continua neste aspecto. Desta forma o método do precursor polimérico tem sido um método muito promissor na preparação de materiais monofásicos, e poucos estudos existem na literatura da síntese de $\mathrm{BiFeO}_{3}$ usando este método. Diante disso, este trabalho apresenta resultados do método de precursor polimérico modificado utilizando o ácido poliacrílico de peso molecular 1800 (PAA1800) como agente quelante (HAN et al., 2006).

\section{MATERIAIS E MÉTODOS}

A síntese dos pós de $\mathrm{BiFeO}_{3}$ foi realizada mediante o método do precursor polimérico modificado, utilizando ácido poliacrílico com peso molecular 1800 (PAA1800) dissolvido em água como agente quelante. Adicionou-se à dissolução de ácido poliacrílico quantidades estequiométricas de $\mathrm{Fe}_{3}(\mathrm{NO})_{3}$ e $\mathrm{Bi}\left(\mathrm{NO}_{3}\right)_{3} \cdot 5 \mathrm{H}_{2} \mathrm{O}$ dissolvidas em $\mathrm{HNO}_{3}$ a $1 \mathrm{M}$. Depois elevouse o pH da solução total em até 9,5 com o objetivo de favorecer a formação do quelato multicatiônico. A solução foi submetida a uma temperatura entre $120{ }^{\circ} \mathrm{C}$ e $140{ }^{\circ} \mathrm{C}$ para a completa evaporação do solvente e a formação da resina polimérica.

A resina resultante foi submetida a analise térmico ATD/TG (Netzsch STA-409), para determinar o comportamento do material em função da temperatura e escolher as temperaturas de calcinação para a obtenção do óxido. A caracterização dos pós cerâmicos obtidos foi realizada por DRX utilizando a linha de luz Síncrotron XRD-1, no Laboratório Nacional de Luz Síncrotron (LNLS) com a descrição experimental reportada por CARVALHO A. M. G. et al. , área de superfície especifica BET (Micromeritics ASAP 2020) e microscopia eletrônica de varredura de alta resolução, FEG XL30.

\section{RESULTADOS E DISCUSSÃO}

A figura 1 apresenta as curvas de ATD/TG da resina polimérica, indicando o efeito da temperatura sobre o solido amorfo obtido. A grande perda de massa que apresenta a amostra entre $300{ }^{\circ} \mathrm{C}$ e $500{ }^{\circ} \mathrm{C}$, está associada principalmente à oxidação da fase orgânica, com a eliminação de $\mathrm{CO}_{2}$, entre $350{ }^{\circ} \mathrm{C}$ e $450{ }^{\circ} \mathrm{C}$ (pico endotérmico). Segundo a curva de ATD, é possível a formação de um oxicarbonato de $\mathrm{Fe}$ e Bi, efeito observado também por (HARDY et al. 2009) para em seguida ocorrer a combustão da fase orgânica com alta taxa de reação, seguido do início da cristalização da fase ferrita de bismuto $\left(\mathrm{BiFeO}_{3}\right)$. 


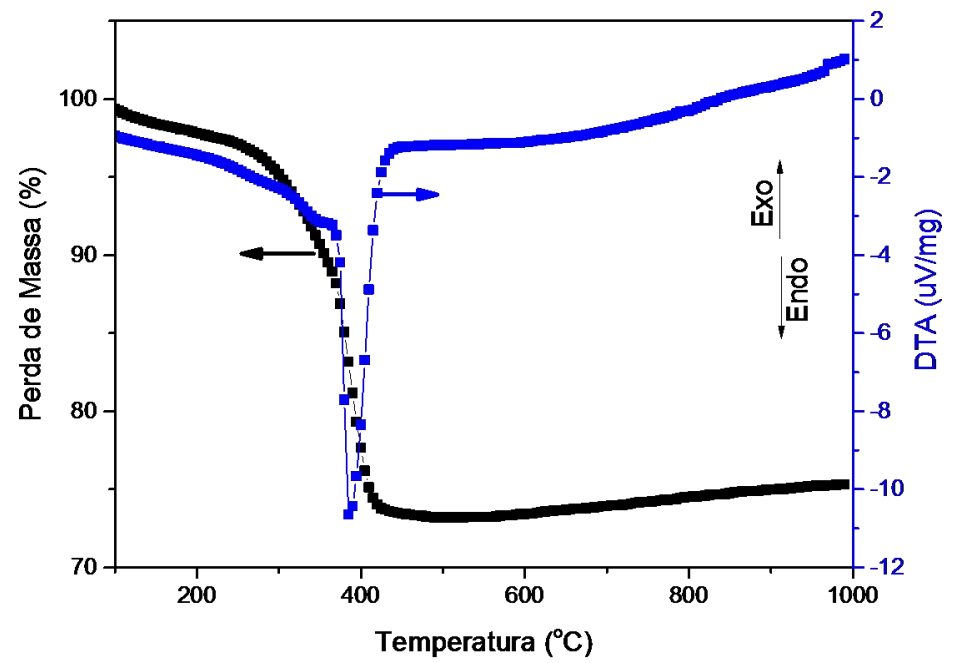

Figura 1. Curvas de $\mathrm{ATD} / \mathrm{TG}$ do sistema $\mathrm{BiFeO}_{3}$ utilizando ácido poliacrílico - PAA1800.

Na figura 2 estão apresentados os perfis de DRX dos pós calcinados a diferentes temperaturas durante 2 horas. A amostra calcinada a $400{ }^{\circ} \mathrm{C}$ não apresentou sinais de fase amorfa, indicando que a completa decomposição da fase orgânica acontece a uma taxa elevada, favorecendo eficientemente a cristalização da $\mathrm{BiFeO}_{3}$ romboédrica (CIF 0107056, PDF 86-1518) junto com a formação simultânea de $\mathrm{Bi}_{25} \mathrm{FeO}_{40}$ (PDF 78-1543) em aproximadamente $22 \%$. A utilização de PAA1 800 reduziu a fase $\mathrm{Bi}_{25} \mathrm{FeO}_{40}$ com o aumento da temperatura de tratamento térmico para $500{ }^{\circ} \mathrm{C}$ e $600{ }^{\circ} \mathrm{C}$, atingindo um valor mínimo de $7 \%$ na amostra tratada termicamente a $600{ }^{\circ} \mathrm{C}$. Em $650{ }^{\circ} \mathrm{C}$ observa-se novamente um aumento considerável da fase $\mathrm{Bi}_{25} \mathrm{FeO}_{40}$ possivelmente pela evaporação de $\mathrm{Bi}_{2} \mathrm{O}_{3}$. Resultados que confirmam a faixa estreita da obtenção de $\mathrm{BiFeO}_{3}$ com o mínimo de fases secundarias termodinamicamente mais estáveis.

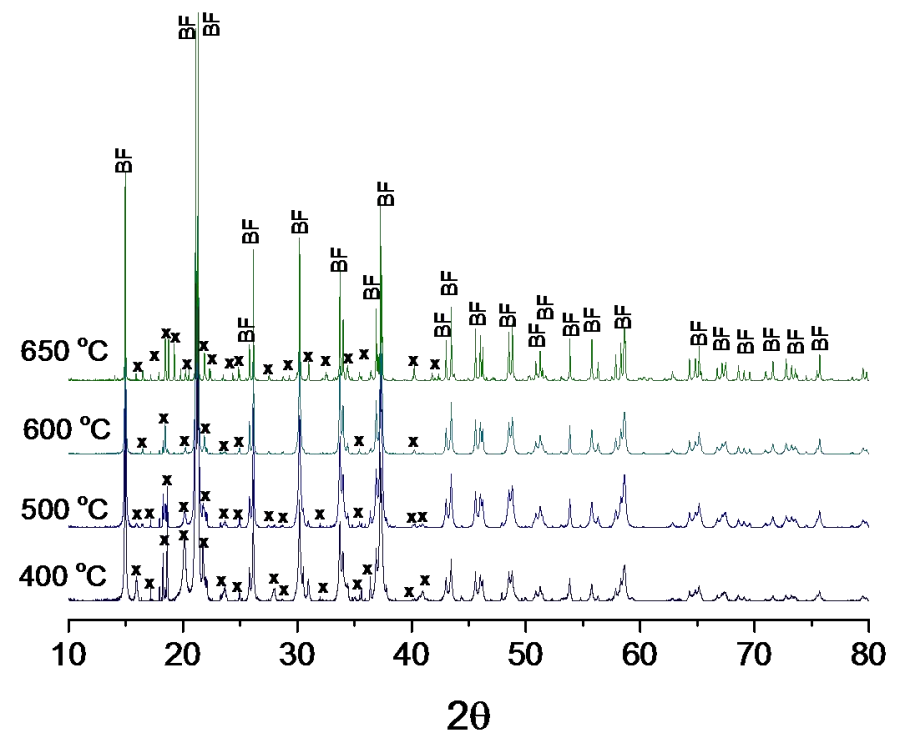

Figura 2- Difratogramas de raios $\mathrm{X}$ dos pós de $\mathrm{BiFeO} 3$ tratados termicamente a diferentes temperaturas durante 2 horas. (BF: $\mathrm{BiFeO} 3$; X: Bi25FeO40). 
Nas imagens de MEV, figura 3, observa-se nos pós calcinados a $400{ }^{\circ} \mathrm{C}$ a formação de aglomerados moles facilmente pulverizáveis, característica típica do método de precursor polimérico. Observa-se também a presença considerável de partículas com morfologia tipo agulha, que podem estar associadas à fase $\mathrm{Bi}_{25} \mathrm{FeO}_{40}$, já que nesta temperatura a amostra sintetizada com o ácido poliacrílico - PAA1 800 apresentou $22 \%$ desta fase, tal como ficou evidenciado pela análise de DRX. Com o aumento da temperatura até $600{ }^{\circ} \mathrm{C}$ observa-se uma morfologia regular das partículas, associada diretamente ao aumento da fase $\mathrm{BiFeO}_{3}$.

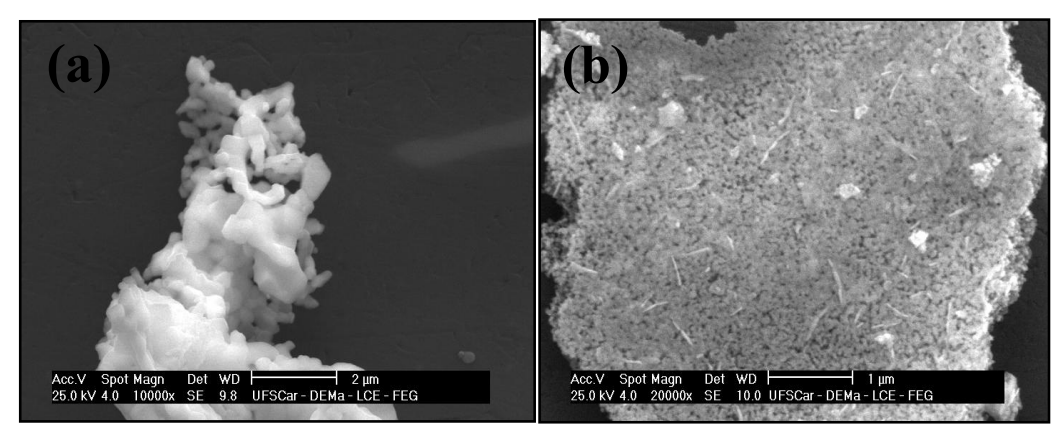

Figura 3- Micrografias MEV da amostra tratada termicamente a (a) $400{ }^{\circ} \mathrm{C} \mathrm{e} \mathrm{(b)} 600{ }^{\circ} \mathrm{C}$.

$\mathrm{Na}$ Tabela I, estão representados os valores de área superficial específica $\left(\mathrm{S}_{\mathrm{BET}}\right)$ dos pós de $\mathrm{BiFeO}_{3}$. Observou-se um aumento gradual da área superficial $\mathrm{S}_{\mathrm{BET}}$ com o aumento da temperatura, como era de se esperar. Os valores de tamanho de cristalito foram estimados mediante a equação de Scherrer, no pico de difração de maior intensidade (plano (310)), mostrando tamanhos de cristalito pequenos, indicando um particulado fino.

Tabela 1 - Valores da área superficial específica, tamanho médio de partículas e tamanho de cristalito da $\mathrm{BiFeO}_{3}$

\begin{tabular}{cccc}
\hline $\begin{array}{c}\text { Temperatura } \\
\text { de calcinação } \\
\left({ }^{\circ} \mathrm{C}\right)\end{array}$ & $\begin{array}{c}\text { Área superficial } \\
\begin{array}{c}\text { SBET } \\
\left(\mathrm{m}^{2} / \mathrm{g}\right)\end{array}\end{array}$ & $\begin{array}{c}\text { Diâmetro } \\
\text { esférico médio } \\
\mathrm{D}_{\mathrm{BET}} \\
(\mathrm{nm})^{*}\end{array}$ & $\begin{array}{c}\text { Tamanho de } \\
\text { cristalito } \\
(\mathrm{nm})^{* *}\end{array}$ \\
\hline 400 & 5,8 & 123,5 & 50 \\
\hline 650 & 3,3 & 213,8 & 70 \\
\hline
\end{tabular}

Nota: * Calculados segundo a equação $D_{B E T}=\frac{6}{D_{t} \cdot S_{R E T}}$ onde $D_{\mathrm{BET}}$ corresponde ao diâmetro esférico equivalente $(\mathrm{nm})$ das partículas, $\mathrm{D}_{\mathrm{t}}$ é a densidade do material $\left(\mathrm{g} / \mathrm{cm}^{3}\right)$ e $\mathrm{S}_{\mathrm{BET}}$ é a área superficial $\left(\mathrm{m}^{2} / \mathrm{g}\right) . * *$ Calculado segundo a equação de Scherrer. 


\section{CONCLUSÃO}

O ácido poliacrílico 1800 utilizado como agente quelante não só favoreceu a obtenção de partículas de $70 \mathrm{~nm}$, após calcinação a $650{ }^{\circ} \mathrm{C}$, como também favoreceu a obtenção da ferrita de bismuto mais pura, com o mínimo de $7 \%$ de fase secundária, conferindo uma alternativa muito promissora na síntese deste material mediante o método do precursor polimérico.

\section{AGRADECIMENTOS}

Os autores agradecem ao Laboratório Nacional de luz sincrotron LNLS e ao Dr. Alexandre M. G. Carvalho pelas analise de raios X na linha de luz XRD1 (projeto: 20160680), à CAPES (PNPD20131474 - 33001014004P9) e FAPESP (2016/22350-1) pelas bolsas concedidas.

\section{REFERÊNCIAS}

CARvalho A. M. G., ARAUjO D. H. C., CANOVA H. F., RODELla C. B., BARRETT D. H., CUFFINIS. L., COSTA R. N. and NUNES R. S. X-ray powder diffraction at the XRD1 beamline at LNLS. Journal of Synchrotron Radiation. 23, p.1501-1506 (2016).

ČEBELAA, M.; ZAGORACA, D.; BATALOVIĆB, K.; RADAKOVIĆB, J.; STOJADINOVIĆC, B.; SPASOJEVIĆD, V.; HERCIGONJAE, R. BiFeO3 perovskites: A multidisciplinary approach to multiferroics. Ceramics International. 43, p. 12561264 (2017).

FISCHER, P.; POLOMSKA, M.; SOSNOWSKA, I. Temperature dependence of the crystal and magnetic structures of BiFeO3. Journal Physics C: Solid State Physics. 13, p. 1931-1940 (1980).

HAN, J. T.; HUANG, Y. H.; WU, X. J.; WEI, W. Tunable synthesis of bismuth ferrites with various morphologies. Advanced Materials. 18, p.2145-2148 (2006).

HARDY A., GIELIS S., VAN DEN RUL H., D’HAEN J., VAN BAEL M.K., MULLENS J. Effects of precursor chemistry and thermal treatment conditions on obtaining phase pure bismuth ferrite from aqueous gel precursors. Journal of the European Ceramic Society 29, p.3007-3013 (2009).

MAITRE, A.; FRANCOIS, M.; GACHON, J. C. Experimental study of the Bi2O3-Fe2O3 pseudobinary system. Journal Phase Equilibria Diffusion. 25, p. 59-67(2004). 